\title{
Gravitational lensing time delays as a tool for testing Lorentz Invariance Violation ${ }^{1}$
}

\author{
Marek Biesiada, Aleksandra Piórkowska \\ University of Silesia, Department of Astrophysics and Cosmology, \\ Uniwersytecka 4, 40-007 Katowice, Poland
}

\begin{abstract}
It is generally expected that quantum gravity theory will bring the picture of a space-time foam at short distances leading to Lorentz Invariance Violation (LIV) manifested e.g. by energy dependent modification of standard relativistic dispersion relation. One direction of research, pursued intensively is to measure energy dependent time of arrival delays in photons emitted by astrophysical sources located at cosmological distances. This is tempered however by our ignorance of intrinsic emission delays in different energy channels.

In this paper we discuss a test based on gravitational lensing. Monitoring time delays between images performed in different energy channels (e.g. optical - low energy and $\mathrm{TeV}$ photons) may reveal extra delays due to distorted dispersion relation typical in LIV theories - a test which is free from the systematics inherent in other settings.
\end{abstract}

Key words: gravitational lensing, quantum gravity phenomenology PACS: 95.30.Sf, 04.60.-m

\section{Introduction}

Despite the fact that quantum gravity theory still remains elusive, it is generally expected that it will bring the picture of a space-time foam at short distances leading to Lorentz Invariance Violation (LIV) manifested e.g. by energy dependent modification of standard relativistic dispersion relation [1,2].

Email addresses: biesiada@us.edu.pl (Marek Biesiada), apiorko@us.edu.pl (Aleksandra Piórkowska).

1 The work was supported by the Polish State Committee for Scientific Research Grant N N203 390034. 
Several years ago it has been proposed to use astrophysical objects to look for energy dependent time of arrival delays [1]. Specifically gamma ray bursts (GRBs) being highly energetic events visible from cosmological distances are the most promising sources of constraining LIV theories [3, 4,5,6]. Among other sources the BL Lac objects like Mk 501 are considered. It is this particular object from which $20 \mathrm{TeV}$ photons were reported [2]. Such objects (also called blazars) have similar nature with quasars. In the papers quoted above some limits on LIV energy scale have been derived (and recently corrected [7]).

The idea of searching for time of flight delays is tempered however by our ignorance of intrinsic delay (at source frame) in different energy channels (see e.g. [4]). Recently it was also shown [8], that lack of detailed knowledge about cosmological model (in the context of accelerating expansion of the Universe) could be another source of systematic effects at high redshifts.

In this paper we discuss a test based on gravitational lensing. Its idea has been noticed in [1] in the context of gamma ray bursts playing the role of high energy photons. Namely such a source located at a cosmological distance may undergo gravitational lensing by a galaxy lying closer to the observer along the line of sight with an encounter parameter small enough to produce multiple images [9] (the so called strong lensing). Indeed all of known strong lensing systems [10] have quasar as a source and a galaxy (in most cases elliptical) as a lens. The light signals emitted by a source will be seen by the observer delayed (achromatically in classical General Relativity) at the location of images. This opens up a possibility to study time delays induced by LIV. Namely, monitoring the time delays between lensed images performed in different energy channels (e.g. optical or gamma-ray — low energy and TeV - high energy photons) may reveal extra delays due to distorted dispersion relation typical in LIV theories. This test is free from the systematics inherent in other settings. Next sections will substantiate the argument further.

\section{LIV induced time delays in different cosmological models}

Following [2] let us consider a phenomenological approach for LIV by assuming the modified dispersion relation for photons in the form:

$$
E^{2}-p^{2} c^{2}=\epsilon E^{2}\left(\frac{E}{\xi_{n} E_{Q G}}\right)^{n}
$$

where: $\epsilon= \pm 1$ is "sign parameter" [2], $\xi_{n}$ is a dimensionless parameter. As a first guess one may assume $E_{Q G}$ equal to the Planck energy, $\xi_{1}=1$ and

$\xi_{2}=10^{-7}[6$. The dispersion relation (11) essentially corresponds to the power- 
law expansion (see [3]) so for practical purposes (due to smallness of expansion parameter $E / E_{Q G}$ ) only the lowest terms of the expansion are relevant. Because in some LIV theories the odd power terms might be forbidden [11] usually the cases of $n=1$ and $n=2$ are retained. It should be noted that in some string theories third order corrections appear as leading ones.

The relation (1) leads to a hamiltonian

$$
\mathcal{H}=\sqrt{p^{2} c^{2}\left[1+\epsilon\left(\frac{E}{\xi_{n} E_{Q G}}\right)^{n}\right]}
$$

from which time dependent group velocity $v(t)=\frac{\partial \mathcal{H}}{\partial p}$ can be inferred.

The comoving distance travelled by photon to the Earth is

$$
r(t)=\int_{t_{\text {emission }}}^{t_{\text {detection }}} v(t) d t=\int_{0}^{z} v\left(z^{\prime}\right) \frac{d z^{\prime}}{H\left(z^{\prime}\right)\left(1+z^{\prime}\right)}
$$

where in the last equation a standard time-redshift parametrization was taken into account. $H(z)$ here denotes the Universe's expansion rate (so called Hubble function). Starting from this point our considerations will have cosmological connotation. The reason for this is simple - the modifications due to LIV theories are really tiny, so one has to look for sources located at cosmological distances (such like quasars or gamma ray bursts) which are far enough to compensate for the smallness of LIV corrections. This means that cosmological background geometry should be taken into account. From now on we will assume it to be a flat Friedmann-Robertson-Walker model with $\Omega_{m}=0.3$ and $\Omega_{\Lambda}=0.7$ - the so called concordance model, as supported by observations [12]. Some alternatives to the concordance model could in principle be discussed as well [8].

Expressing group velocity in terms of redshift, we get

$$
v(z) \simeq c(1+z)\left[1+\epsilon \frac{(n+1)}{2}\left(\frac{E}{\xi_{n} E_{Q G}}\right)^{n}(1+z)^{n}\right]
$$

Time of flight for the photon of energy $E$ is equal to

$$
t_{L I V}=\int_{0}^{z}\left[1+\epsilon \frac{n+1}{2}\left(\frac{E}{\xi_{n} E_{Q G}}\right)^{n}\left(1+z^{\prime}\right)^{n}\right] \frac{d z^{\prime}}{H\left(z^{\prime}\right)}
$$

In the first term one easily recognizes the time of flight for photons in standard relativistic cosmology (i.e. without LIV). Due to very small magnitude of LIV 
corrections it also fairly represents the time of flight for low energy photons. Therefore below we neglect LIV corrections at low energy.

Consequently, the time delay between a low energy and a high energy photon is equal to

$$
\Delta t_{L I V}=\frac{n+1}{2}\left(\frac{E}{\xi_{n} E_{Q G}}\right)^{n} \int_{0}^{z} \frac{\left(1+z^{\prime}\right)^{n} d z^{\prime}}{H\left(z^{\prime}\right)}
$$

where we restricted our attention to "infraluminal" motion of high energy photons (i.e. low energy photons arrive earlier to the observer). Generalization to "superluminal" motion is straightforward - the same value with just an opposite sign (time delays become early arrivals).

The idea of observational strategy emerging form (6) is again simple: monitor appropriate (i.e. emitting both low and high energy photons) cosmological source at different energy channels and try to detect this time delay. Some attempts along this line have already been undertaken [3,4,13]. However there remains an indispensable uncertainty about intrinsic time delays: there is no reason for which low and high energy signal should be emitted simultaneously, and while detecting distinct signals (peaks in the light curve) at different energies we have no idea which one was sent first.

Our method outlined below, invoking gravitational lensing allows to get rid of this ambiguity. Before describing it in subsequent paragraph let us recall now that in cosmology one distinguishes three types of distances:

(i) comoving distance:

$$
r(z)=c \int_{0}^{z} \frac{d z^{\prime}}{H\left(z^{\prime}\right)}=\frac{c}{H_{0}} \tilde{r}(z)
$$

by $\tilde{r}(z)$ we denoted a reduced (dimensionless) comoving distance, i.e. a comoving distance expressed as a fraction of the Hubble horizon $d_{H}=c / H_{0}$,

(ii) angular diameter distance:

$$
D_{A}(z)=\frac{1}{1+z} r(z)
$$

(iii) luminosity distance:

$$
D_{L}(z)=(1+z) r(z)
$$


Angular diameter distance is the one used in gravitational lensing theory (because gravitational lensing deals with light deflection i.e. essentially with angles). The luminosity distance is a measure invoked while using standard candles (e.g. SNIa). The point is that both distance measures are related to the comoving distance by a $1+z$ factor (see above). The comoving distance, then, is closely related to the time of flight $r(z)=c t$. It is in fact the distance to the source measured in light years. Therefore we can rewrite the time of flight in LIV theory (5) in terms of comoving distance: $r_{L I V}(z)=c t_{L I V}$. Of course the comoving distance to the source is fixed - there are photons of different energies that travel with different speed. It is however useful (for later calculations) to think as if they travelled with the same speed $c$ but along different comoving distances $r(z)$ and $r_{L I V}(z)$.

\section{Gravitational lensing time delays}

Gravitational lensing of quasars and extragalactic radio sources at high redshifts by foreground galaxies is now well established and has developed into a mature branch of both theoretical and observational astrophysics [9]. Misalignment of the source, the lens and observer results typically in multiple images whose angular positions and magnification ratios allow reconstructing lensing mass distribution. In particular they provide an independent confirmation of dark matter in galaxies and became an important tool for investigating dark matter distribution. Another important ingredient of gravitational lensing is the time delay between lensed images of the source. This effect originates as a competition between Shapiro time delay from the gravitational field and the geometric delay due to bending the light rays and is best understood in terms of Fermat principle. In other words, the intervening mass between the source and the observer introduces an effective index of refraction, thereby increasing the light travel time.

In general, the light travel time can be calculated as

$$
t(\vec{x})=\frac{1+z_{l}}{c} \frac{D_{l} D_{s}}{D_{l s}}\left[\frac{1}{2}(\vec{x}-\vec{\beta})^{2}-\psi(\vec{x})\right]
$$

where: $\vec{x}$ and $\vec{\beta}$ are positions (as projected on the celestial sphere) of the image and the source, $\psi(\vec{x})$ is the projected gravitational potential (i.e. the actual potential integrated along line of sight), $D_{l}, D_{s}$ are angular diameter distances to the lens and the source located at redshifts $z_{l}$ and $z_{s}$ respectively ( $D_{l s}$ is the angular diameter distance between lens and source). From now on we adopt the notation (standard in gravitational lensing theory) where $D$

denotes angular diameter distance, and subscripts refer to the components of 
lensing system (i.e. the source, the lens or the observer).

The lensing is called strong if source position happens to lie within the so called Einstein ring — the circle of a radius $\vartheta_{E}$ (defining the proper deflection scale of a given lens). In this case multiple images appear and since lensing galaxies are often ellipticals, the number of images is usually equal to four [10,14] (or five [15] - the issue of image multiplicity is discussed e.g. in [9]). However, the surprisingly realistic model of the lens potential is that of a singular isothermal sphere (SIS) [9]. Indeed lensing by elipticals can be modelled by its variant called singular isothermal ellipsoid (SIE). Therefore for the purpose of illustrating our ideas we shall restrict our attention to the SIS model since generalization to SIE is rather straightforward and would not change our conclusions.

The Einstein ring radius for the SIS model is:

$$
\vartheta_{E}=4 \pi \frac{D_{l s}}{D_{s}} \frac{\sigma^{2}}{c^{2}}
$$

where $\sigma$ denotes one-dimensional velocity dispersion of stars in lensing galaxy. If the lensing is strong i.e. $\beta:=|\vec{\beta}|<\vartheta_{E}$ then two co-linear images $\mathrm{A}$ and $\mathrm{B}$ form on the opposite side of the lens, at radial distances $R_{A}=\beta+\vartheta_{E}$ and $R_{B}=\vartheta_{E}-\beta$ having time delays between the images:

$$
\Delta t_{S I S}=\frac{1+z_{l}}{2 c} \frac{D_{l} D_{s}}{D_{l s}}\left(R_{A}^{2}-R_{B}^{2}\right)
$$

which according to the above mentioned relations for SIS model can also be written as

$$
\Delta t_{S I S}=\frac{2\left(1+z_{l}\right)}{c} \frac{D_{l} D_{s}}{D_{l s}} \vartheta_{E} \beta=\frac{8 \pi}{H_{0}} \widetilde{r}_{l} \beta \frac{\sigma^{2}}{c^{2}}
$$

In the last equation $\tilde{r}_{l}$ denotes the reduced comoving distance to the lens. The equation (12) is commonly used by gravitational lensing community because it reduces time delay problem to relative astrometry of images, whereas $\beta$ is much harder to asses (it is small in order for strong lensing to occur) and Einstein ring radius is not a directly observable quantity. However, the equation (13) is more useful from the theoretical point of view. In particular it shows explicitly that the time delay between images is created at the lens location ( $\widetilde{r}_{l}$ factor). Let us stress again that this time delay is achromatic in General Relativity. 


\section{LIV induced time delays and gravitational lensing time delays}

Let us now imagine a source at cosmological distance emitting low energy and high energy (in $\mathrm{TeV}$ range) photons which undergoes gravitational lensing by a foreground galaxy. Let us also assume that LIV type distorted dispersion relation (1) holds in nature. The observer would notice again time delays between images, but this time it would be a combined effect of gravitational lensing and LIV. Therefore it would no longer be achromatic. This idea was formulated originally in [1] but up to our best knowledge it has not been further developed.

It is rather straightforward to calculate this by using the above mentioned fictitious "LIV comoving distance" $r_{L I V}(z)$, namely:

$$
\Delta t_{L I V, S I S}=\frac{8 \pi}{H_{0}} \widetilde{r}_{L I V}\left(z_{l}\right) \beta \frac{\sigma^{2}}{c^{2}}
$$

where:

$$
\widetilde{r}_{L I V}\left(z_{l}\right)=\widetilde{r}_{l}+H_{0} \frac{n+1}{2}\left(\frac{E}{\xi_{n} E_{Q G}}\right)^{n} \int_{0}^{z_{l}} \frac{\left(1+z^{\prime}\right)^{n} d z^{\prime}}{H\left(z^{\prime}\right)}
$$

Because the LIV effect is extremely small, let us restrict further to the $n=1$ case:

$$
\widetilde{r}_{L I V}\left(z_{l}\right)=\widetilde{r}_{l}+H_{0} \frac{E}{E_{Q G}} \int_{0}^{z_{l}} \frac{\left(1+z^{\prime}\right) d z^{\prime}}{H\left(z^{\prime}\right)}
$$

Now we can assume that observations in low energy would essentially provide time delay between images equal to $\Delta t_{S I S}$, whereas monitoring of the same images in high energy $(\mathrm{TeV})$ channel would provide $\Delta t_{\text {LIV,SIS }}$. These two measurements would differ by

$$
\Delta t_{L I V, S I S}-\Delta t_{S I S}=\frac{8 \pi}{H_{0}} \beta \frac{\sigma^{2}}{c^{2}} \frac{E}{E_{Q G}} \int_{0}^{z} \frac{\left(1+z^{\prime}\right) d z^{\prime}}{H\left(z^{\prime}\right)}
$$

Rigorously one should rather calculate $\Delta t_{L I V, S I S}\left(E_{1}\right)-\Delta t_{L I V, S I S}\left(E_{2}\right)$ and write $\Delta E=E_{1}-E_{2}$ in the numerator of (17) but due to our assumption that $E_{2}$ is many orders of magnitude smaller than $E_{1}$ and thus having negligible LIV correction the above expression is a good appproximation. Let us make an estimate for the above LIV effect taking a real strong lensing system. HST $14176+5226$ can serve as an example. This system was discovered with the 
Hubble Space Telescope [14] and further confirmed to be a gravitational lens [16]. The lensed source is a quasar at redshift $z_{s}=3.4$ whereas the lens is an elliptical galaxy having redshift $z_{l}=0.809$. The lens model best fitted to the observed images was based on a singular isothermal ellipsoid [17] giving the Einstein radius $\theta_{E}=1^{\prime \prime} .489$ and $\beta=0 " .13=8.4 \times 10^{-7} \mathrm{rad}$.

Optical spectroscopy of the lensing galaxy in HST $14176+5226$ system [18] provided measurements of the velocity dispersion in lensing galaxy. These measurements have been confirmed by Treu and Koopmans [19] who performed spectroscopic observations on Keck telescope as part of Lenses Structure and Dynamics (LSD) Survey. The result is $\sigma=290 \pm 8 \mathrm{~km} / \mathrm{s}$.

Substituting these data to (17) gives $\Delta t_{L I V, S I S}-\Delta t_{S I S}$ equal to $3.7 \times 10^{-9} \mathrm{~s}$ for $5 \mathrm{TeV}$ photons and $1.5 \times 10^{-8} \mathrm{~s}$ for $20 \mathrm{TeV}$ ones.

The model presented above was the simplest one just because its aim was to illustrate ideas. So was also the purpose of choosing the HST 14176+5226 system as an example. In reality one would encounter systems with different numbers of images or different separations, e.g. SDSS J1004+4112 [20] with an image separation of 14 ". 6 or recently discovered system SDSS J1029+2623, where a quasar at $z_{s}=2.197$ is doubly imaged by a massive galaxy cluster at $z_{l}=0.55$ with images separation of $22 " .5$ [21].

However none of these effects is crucial to our general arguments because of their differential setting ( $\Delta t_{\text {high energy }}$ vs. $\left.\Delta t_{\text {low energy }}\right)$. High and low energy photons from each image travel along the same paths (respectively) thus suffering the same shear effects (respectively). We do not mean here that one could disregard these effects but rather to strengthen the standard lore of lensing community to treat each lensing system separately in a detailed manner.

Closing this section it would be interesting to ask how the LIV effects might modify image configurations. It could be suspected that they might do so since from the Fermat's principle perspective images are located at stationary points of the wavefront travel time functional (given by equation (10)). Therefore since LIV modifies time of flight in an energy dependent way (due to modified dispersion relation) then one expects the images seen at different energies located at different positions. It is easy to see that for the SIS lens (generalizations to other mass profiles are also rather straightforward) the difference between Einstein radii for high and low energy photons $\Delta \theta_{E, L I V}:=\theta_{E, L I V}-\theta_{E}$ would be given by formula:

$$
\Delta \theta_{E, L I V}=\theta_{E} \frac{E}{E_{Q G}}\left(\frac{I^{(1)}\left(z_{l}, z_{s}\right)}{\widetilde{r}\left(z_{l}, z_{s}\right)}-\frac{I^{(1)}\left(z_{s}\right)}{\widetilde{r}\left(z_{s}\right)}\right)
$$

where: $I^{(1)}\left(z_{1}, z_{2}\right):=\int_{z_{1}}^{z_{2}} \frac{\left(1+z^{\prime}\right) d z^{\prime}}{H\left(z^{\prime}\right)}$. For realistic lens configurations like HST 
$14176+5226$ this would give negligibly small corrections of order $10^{-16}$ arc sec. Hence even if LIV were operating this would not be able to change macroimages position in a detectable way. However it cannot be excluded that such minute differences could become relevant while studying caustic crossing possibly leading to different magnification patterns due to microlensing at different energies.

\section{Discussion and conclusions}

In this paper we discussed a method (first noticed in [1]) to test LIV effects by monitoring time delays between images of gravitationally lensed quasars in low and high energy channels. In standard theory (General Relativity) the result should be the same - gravitational lensing is essentially achromatic. On the other hand in the presence of LIV effects time delays loose this property - high energy photons should come at different times comparing with low energy ones. Therefore time delays between images should be different at different energies (e.g. optical or gamma-rays and $\mathrm{TeV}$ photons). We are tacitly assuming (following approach taken by the rest of LIV studying community) that LIV effects are manifested only in high energy domain (where the small scale "foamy" structure of the space-time reveals itself) whereas the overall background geometry of space-time shaped by low energy content of the Universe is that of General Relativity (more precisely - the flat Friedman-Robertson-Walker model as suggested by cosmological data) with light deflection (i.e. geodesic motions) defined in a standard way.

Because this method is differential in nature, it gets rid of the assumptions about intrinsic time delays of signals at different energies. In fact time delays between images at different energies could be established in different experiments (at unrelated observing sessions) performed on given lensing system. The only demand is that they are accurate enough (done with a sufficient temporal resolution). Since the time delay between images is produced at the lens location, the result does not depend very strongly on the cosmological model. Lenses are located at modest redshifts where all realistic cosmological models essentially agree.

One may ask if appropriate lensing systems (i.e. having sources emitting both low and high energy photons) exist. It is an observational fact that very high energy emission $(E>100 \mathrm{GeV})$ has been detected from over a dozen of blazars [22] which have similar nature with quasars. Quasars, on the other hand are the sources in all known strong lensing systems - CASTLES database contains a 100 of such systems [10]. It is a matter of coordinating strong lensing surveys with experiments in high energy astrophysics (such like AGILE, GLAST or

MAGIC experiments [23]) and the future will certainly bring the discovery of 
lensed high energy source. Angular resolution of high energy experiments is gradually being improved. For example a recently launched AGILE instrument [24] has been designed to obtain accurate localization $\left(\sim 2^{\prime}-3^{\prime}\right)$ of transient events by the Gamma Ray Imaging Detector (GRID) - SA combination. So it is already close to typical image separation and definitely being able to see images like those from SDSS J1004+4112 or SDSS J1029+2623 as separated. Depending on exposure and the diffuse background its flux sensitivity threshold can reach values of $(10-20) \times 10^{-8}$ photons $\mathrm{cm}^{-2} \mathrm{~s}^{-1}$ at energies higher than $100 \mathrm{MeV}$ with with an effective area above $200 \mathrm{~cm}^{2}$ at $30 \mathrm{MeV}$. Moreover it has excellent timing capability, with overall photon absolute time tagging of uncertainty below $2 \mu s$ and very small deadtimes $(<200 \mu s$ for the GRID, $\sim 5 \mu \mathrm{s}$ for the sum of the SA readout units, and $\sim 20 \mu s$ for each of the individual CsI bars). In fact, AGILE instrument is optimized in the range below $1 \mathrm{GeV}$ hence it is not representative to $\mathrm{TeV}$ range experiments needed to probe LIV but it clearly shows the the gradual improvement of sensitivity, timing and angular resolution in high energy astrophysics.

An order of magnitude estimate for the effect discussed in this paper is not encouraging now. For a typical lensing system like HST $14176+5226$ it is of order of nano-seconds. However, having in mind that high energy astrophysical sources display rapid variability (indeed intrinsic variability in relativistic shocks powering these sources is enhanced by a Lorentz factor typically of order of $10^{2}$ ) and that e.g. light curves of gamma-ray bursts are already sampled with mili-second resolution (and AGILE went down to micro-seconds) one should not reject the idea presented above on the grounds that it is not within the scope of present-day observational technology.

\section{References}

[1] G. Amelino-Camelia, J.R. Ellis, N.E. Mavromatos, D.V. Nanopoulos and S. Sarkar, Nature 393, 763 (1998).

[2] G. Amelino-Camelia and T. Piran, Phys. Rev. D 64, 763 (2001).

[3] J.R. Ellis, N.E. Mavromatos, D.V. Nanopoulos and A.S. Sakharov, Astron. Astrophys. 402, 409 (2003);

S.E. Boggs, C.B. Wunderer, K. Hurley and W. Coburn, ApJ 611, L77-L80 (2004).

[4] J. Ellis, N.E. Mavromatos, D.V. Nanopoulos, A.S. Sakharov and E.K.G. Sarkisyan, Astropart.Phys. 25 402-411 (2006), arXiv:astro-ph/0510172.

[5] M. Rodriguez Martinez and T. Piran, J. Cosmol. Astropart. Phys. JCAP04(2006)006 (2006).

[6] U. Jacob and T. Piran, Nature Phys. 387 (2007). 
[7] U. Jacob and T. Piran, (2007), arXiv:0712.2170;

J.R. Ellis, N.E. Mavromatos, D.V. Nanopoulos, A.S. Sakharov and E.K.G. Sarkisyan, arXiv:0712.2781.

[8] M. Biesiada and A. Piórkowska, J. Cosmol. Astropart. Phys. JCAP05(2007)011 (2007), arXiv:0712.0937.

[9] P. Schneider, J. Ehlers and E.E. Falco, Gravitational Lenses, (Springer Verlag, Berlin, 1992).

[10] CASTLES web page: http://cfa-www.harvard.edu/castles/

[11] C.P. Burgess, J.M. Cline, E. Filotas, J. Matias and G.D. Moore, J. High Energy Phys. JHEP03(2002)043 (2002).

[12] D. Spergel et al., Astrophys. J Suppl. 148, 175 (2003).

[13] J. Albert et al., Probing Quantum Gravity using Photons from a Mkn 501 Flare Observed by MAGIC, (2007), arXiv: 0708.2889.

[14] K.U. Ratnatunga, E.J. Ostrander and R.E. Griffiths, Astrophys. J. 453, L5 (1995).

[15] N. Inada et al., Publ.Astron.Soc.Jap. 57, L7 (2005).

[16] D. Crampton, O. Le Fevre, F. Hammer and S.J. Lilly, Astron. Astrophys. 307, L53 (1996).

[17] K.U. Ratnatunga, R.E. Griffiths and E.J. Ostrander, Astron. J. 117, 2010 (1999).

[18] T. Ohyama et al., Astron. J. 123, 2903 (2002).

[19] T. Treu and L.V.E. Koopmans, Astrophys. J. 611, 739 (2004).

[20] N. Inada et al., Nature 426, 810 (2003).

[21] N. Inada et al., Astrophys.J. 653, L97 (2006).

[22] R. Wagner, Proc. 30th International Cosmic Ray Conference, arXiv:0706.4442,

[23] M. Persic, A. De Angelis, F. Longo and M. Tavani, Gamma-ray blazars: the combined AGILE and MAGIC views, Proc. 30th International Cosmic Ray Conference, arXiv:0709.1881v1.

[24] M. Tavani et al., AGILE in orbit, Proc. 5th International Workshop "Science with the New Generation of High Energy Experiments", eds. A. Lionetto and A. Morselli, (Frascati Physics Series, 2007), Vol.XLV, p.1. 